\title{
FORMATION OF GALACTIC SYSTEMS IN LIGHT OF THE MAGNESIUM ABUNDANCE IN FIELD STARS.III.THE HALO
}

\author{
V. A. Marsakov, T. V. Borkova \\ Institute of Physics, Rostov State University, \\ 194, Stachki street, Rostov-on-Don, Russia, 344090 \\ e-mail: marsakov@ip.rsu.ru, borkova@ip.rsu.ru \\ accepted 2006, Astronomy Letters, Vol. 32 No. 8, P.545-556
}

\begin{abstract}
Data from our compiled catalog of spectroscopically determined magnesium abundances in dwarfs and subgiants with accurate parallaxes are used to select Galactic halo stars according to kinematic criteria and to identify presumably accreted stars among them. Accreted stars are shown to constitute the majority in the Galactic halo. They came into the Galaxy from disrupted dwarf satellite galaxies. We analyze the relations between the relative magnesium abundances, metallicities, and Galactic orbital elements for protodisk and accreted halo stars. We show that the relative magnesium abundances in protodisk halo stars are virtually independent of metallicity and lie within a fairly narrow range while presumably accreted stars demonstrate a large spread in relative magnesium abundances up to negative $[\mathrm{Mg} / \mathrm{Fe}]$. This behavior of protodisk halo stars suggests that the interstellar matter in the early Galaxy mixed well at the halo formation phase. The mean metallicity of magnesium-poor $([\mathrm{Mg} / \mathrm{Fe}]<0.2 \mathrm{dex})$ accreted stars has been found to be displaced toward the negative values when passing from stars with low azimuthal velocities $\left(|\Theta|<50 \mathrm{~km} \mathrm{~s}^{-1}\right)$ to those with high ones at $\Delta[\mathrm{Fe} / \mathrm{H}] \approx 0.5 \mathrm{dex}$. The mean apogalactic radii and inclinations of the orbits also increase with increasing absolute value of $|\Theta|$ while their eccentricities decrease. As a result negative radial and vertical gradients in relative magnesium abundances are observed in the accreted halo in the absence of correlations between the $[\mathrm{Mg} / \mathrm{Fe}]$ ratios and other orbital elements, while these correlations are found at a high significance level for genetically related Galactic stars. Based on the above properties of accreted stars and our additional arguments, we surmise that as the masses of dwarf galaxies decrease, the maximum SN II masses and hence, the yield of $\alpha$-elements in them also decrease. In this case, the relation between the $[\mathrm{Mg} / \mathrm{Fe}]$ ratios and the inclinations and sizes of the orbits of accreted stars is in complete agreement with numerical simulations of dynamical processes during the interaction of galaxies. Thus the behavior of the magnesium abundance in accreted stars suggests that the satellite galaxies are disrupted and lose their stars en masse only after dynamical friction reduces significantly the sizes of their orbits and drags them into the Galactic plane. Less massive satellite galaxies are disrupted even before their orbits change appreciably under tidal forces.
\end{abstract}

Keywords: Galaxy (Milky Way), stellar chemical composition, accreted stars, halo, Galactic evolution. 


\section{Introduction}

A detailed analysis of the chemical composition of the metal-poorest field stars, together with their Galactic orbital elements, makes it possible to reconstruct the formation history of the halo in the early Galaxy. Metal-poor stars are currently believed to form at least two subsystems, an accreted halo and a protodisk halo. The first subsystem was formed by the stars and globular clusters captured by the Galaxy at different times from debris of dwarf satellite galaxies disrupted by its tidal forces. The stars of the second subsystem, along with the metal-richer Galactic stars, were formed mainly from the matter of a single protogalactic cloud. It is unlikely that the interstellar matter from which the stars of these two metal-poor subsystems were formed has experienced an exactly coincident chemical evolution. Therefore, it would be interesting to search for subtle differences between them that could shed light on the histories of star formation and matter mixing inside and outside the single protogalactic cloud. Owing to the favorable "geographic" position of the Sun in the Galactic plane, we have an opportunity to observe the stars of all its subsystems in the immediate vicinity of the Sun and to analyze in detail their chemical composition. Indeed even the stars that rise high above the Galactic plane cross this plane twice during one revolution around the Galactic center and, if the perigalactic radii of their orbits are smaller than the solar one they sooner or later come close to the Sun. In addition it is interesting to identify stars with identical current Galactic orbits (i.e., those that presumably have a common origin) in the accreted halo and to try to trace the pattern of variations in the relative abundance, e.g., of $\alpha$-elements with increasing metallicity for them. If a clearly traceable relation is found between these parameters this, on the one hand will serve as further evidence for their genetic relationship and on the other hand will make it possible to compare the star formation rates and the patterns of chemical evolution in this group and in the early Galaxy.

The stars that currently belong to the protodisk halo were formed over a fairly short period. This follows from the fact that the firmly established earliest traces of enrichment of the interstellar medium by type Ia supernova (SN Ia) ejecta are observed only in the stars of the later formed thick-disk subsystem (Mashonkina and Gehren 2001; Marsakov and Borkova 2005). The evolution time of close binary stars that subsequently explode as SNe Ia is short, 0.5-1.5 Gyr (see, e.g., Matteucci 2001; Tsujimoto et al. 1995). Exclusively higher-mass $\left(M>10 M_{\odot}\right)$ stars exploding as type II supernovae (SNe II) are currently believed to have enriched the interstellar medium with heavy elements at earlier stages. Their characteristic evolution time is only $\approx 30$ Myr. Almost all of the nuclei of $\alpha$ elements are formed in SNeII. Concurrently a small amount of iron-peak elements is also produced in these SNe while the bulk of them is ejected into the interstellar space during SN Ia explosions. Calculations show that the yield of various elements in SNe II depends on several parameters. In particular the yield of the socalled primary $\alpha$-elements $(\mathrm{O}$, $\mathrm{Ne}$, and $\mathrm{Mg}$ ) being synthesized in hydrostatic processes in a carbon core and explosive $\mathrm{Ne} / \mathrm{C}$ burning in a presupernova's shell sources depends strongly on the stellar mass (see, e.g., Thielemann et al. 1996; Nomoto et al. 1997). In contrast, the amount of iron-group elements depends on an explosion mechanism related to the iron core size. Therefore, the relative abundances of the primary $\alpha$-elements $([\alpha / \mathrm{Fe}])$ in the ejecta of different SNe II even with the same mass can differ markedly. Fortunately the next generation of stars is born in an interstellar medium that was generally enriched by several supernovae and mixing partially levels off the dependence of the yield on their explosion parameters. Hence, the variations in the upper boundary of the initial mass function for stars that exploded at 
different times inside and outside the early Galaxy can be estimated from the relative abundances of various elements in genetically related stars. Concurrently because of the difference between the evolution times of SNeII and SNeI we can try to trace the star formation rate for this stellar ensemble by the coordinates of the characteristic knee in its $[\alpha / \mathrm{Fe}]-[\mathrm{Fe} / \mathrm{H}]$ diagram toward the sharp decrease in the relative abundance of the primary $\alpha$-elements with increasing total heavy-element abundance at the onset of SNe Ia explosions, i.e., $\sim 1$ Gyr later.

The best-studied primary $\alpha$-elements are oxygen and magnesium because they exhibit several absorption lines in the visible spectral range. Although the theory predicts a dependence of the $\mathrm{Mg}$ yield (in contrast to oxygen) on mixing parameters in the presupernova's atmosphere, we preferred to use here precisely this element, since its abundance in stars is determined much more reliably than that of oxygen. For the analysis, we took data from our compiled catalog of spectroscopically determined magnesium abundances (Borkova and Marsakov 2005). Almost all of the magnesium abundances in dwarfs and subgiants in the solar neighborhood determined by synthetic modeling of high-dispersion spectra published before January 2004 were gathered in this catalog. This catalog is several times larger than any homogeneous sample that has been used until now to analyze the Galactic chemical evolution. The relative magnesium abundances in the catalog were derived from 1412 spectroscopic determinations in 31 publications for 867 dwarfs and subgiants using a three-pass iterative averaging procedure with a weight assigned to each primary source and each individual determination. The internal accuracy of the catalogued relative magnesium abundances for metal-poor $([\mathrm{Fe} / \mathrm{H}]<-1.0 \mathrm{dex})$ stars is $\varepsilon[\mathrm{Mg} / \mathrm{Fe}]= \pm 0.07$ dex. The metallicities for the stars were obtained by averaging about 2000 determinations from 80 publications; the accuracy for metal-poor stars was estimated to be $\varepsilon[\mathrm{Fe} / \mathrm{H}]= \pm 0.13$ dex. The distances to the stars and their space velocities were calculated on the basis of data from currently available high-precision catalogs. We used trigonometric parallaxes with errors smaller than $25 \%$ and, if these were lacking, photometric distances calculated from $u_{v b y H_{\beta}}$ photometry. Based on a multicomponent model of the Galaxy (Allen and Santillan 1991) containing a disk a bulge, and an extended massive halo, we calculated the Galactic orbital elements by simulating 30 revolutions of a star around the Galactic center. The Galactocentric distance of the Sun was assumed to be $8.5 \mathrm{kpc}$, the rotational velocity of the Galaxy at the solar Galactocentric distance was $220 \mathrm{~km} \mathrm{~s}^{-1}$ and the velocity of the Sun with respect to the local standard of rest was $\left(U_{\odot}, V_{\odot}, W_{\odot}\right)=(-11,14,7.5) \mathrm{km} \mathrm{s}^{-1}$ (Ratnatunga et al. 1989).

Previously we analyzed the relative abundances of magnesium and europium (an $\mathrm{r}$ process element) in 77 nearby stars, of which only 23 stars belong to the Galactic halo (Borkova and Marsakov 2004). All of the presumably accreted stars that we identi ed according to kinematic criteria were found to exhibit $[\mathrm{Mg} / \mathrm{Eu}]$ ratios that differ sharply from those in the stars genetically related to the Galaxy. (These differences serve as a further argument in favor of their extragalactic origin.) Analysis of the deviations led us to conclude that the maximum masses of the very first stars exploded as SNe II outside the Galaxy were much lower than those inside it. In contrast, the supernovae exploded outside the Galaxy somewhat later turned out to be more massive than those in the Galaxy itself. This intermediate $[\mathrm{Mg} / \mathrm{Eu}]$ ratio in the metal-poor stars of the early Galaxy may be indicative of more intense mixing inside the single protogalactic cloud.

This paper completes our systematic description of the chemical and spatial-kinematic properties of the stars that are currently in the solar neighborhood but that belong to different Galactic subsystems based on data from our compiled catalog. In our previous papers, 
we considered the properties of thick-and thin-disk stars (see Marsakov and Borkova (2005 and 2006), respectively).

\section{IDENTIFICATION OF STARS OF THE HALO SUBSYS- TEMS}

In our paper devoted to the thick disk, we justified the choice of the residual stellar velocity relative to the local standard of rest res $V_{\text {res }}=175 \mathrm{~km} \mathrm{~s}^{-1}$ as a criterion for separating the thick-disk and halo stars. We optimized the specific value of this criterion by minimizing the number of metal-rich $([\mathrm{Fe} / \mathrm{H}]>-1.0)$ stars of our sample in the identified halo subsystem and metal-poor stars in the thick disk. The need for this optimization procedure is dictated by the presence of adistinctde cit of high-velocity $\left(V_{\text {res }}>100 \mathrm{~km} \mathrm{~s}^{-1}\right)$ stars near this point in their metallicity distribution suggesting the absence of a smooth transition between the halo and the thick disk (see Marsakov and Suchkov 1977).

In identifying the stars of an extragalactic origin (which were called here accreted stars), we assumed that the stars born in a monotonically collapsing single protogalactic cloud could not be in retrograde orbits. In this case, all of the stars with retrograde orbits around the Galactic center may be considered accreted ones. However as can be seen from the spatial distribution of presumably accreted globular clusters (see Fig. 6 in Borkova and Marsakov (2000)), not only the orbits of their parent satellite galaxies differed significantly before their disruption. Therefore we included all of the stars with the same high residual velocities as those for the retrograde ones, i.e., $V_{\text {res }}>240 \mathrm{~km} \mathrm{~s}^{-1}$ in the group of presumably accreted stars. As we see from Fig. 1a, it is at this critical residual velocity that stars in retrograde orbits appear in our sample. We also included two more slower stars (HD 103723 and HD 105004)is this group. (As will be shown below they both simultaneously exhibit high metallicities $([\mathrm{Fe} / \mathrm{H}] \approx-0.73)$ and anomalously low magnesium abundances $([\mathrm{Mg} / \mathrm{Fe}]<0.1)$, which are a typical of the stars of the protodisk halo where they fell according to the residual velocity criterion: $V_{\text {res }}=211$ and $217 \mathrm{~km} \mathrm{~s}^{-1}$ respectively.) Figure $1 \mathrm{~b}$ shows that the spread in apogalactic radii of the stellar orbits increases sharply as one passes through the critical residual velocity; the orbits for stars with prograde motions have even systematically larger sizes than those for stars with the same residual velocity relative to the local standard of rest, but with retrograde motions.

We emphasize that, based only on a kinematic criterion, we cannot identify the stars of a particular Galactic subsystem with absolute confidence. However since the main goal of this paper is to study the abundance differences between some of the chemical elements in stars of the two halo subsystems, we decided to use this chemical-composition independent parameter lest an artificial selection be introduced. Therefore, the personal identification of each star should be considered to be preliminary and to require a further refinement. However this criterion is quite appropriate in statistical analyses to reveal any relations between chemical composition and other parameters of stars of different Galactic subsystems.

\section{RELATION BETWEEN THE IRON AND MAGNESIUM ABUNDANCES}

Thus our kinematic criteria identified a total of about 20 protodisk halo stars, while more than a hundred stars from our sample belong to the accreted halo. By the "protodisk" halo 
we mean the subsystem that consists of stellar objects that are the first to be formed from the matter of a collapsing single protogalactic cloud and that are genetically related to the stars of all the subsequent generations. The appreciably increasing number of genetically related stars with metallicity (see Fig. 2) suggests that more active star formation in the early Galaxy began slightly later when the mean metallicity of the interstellar medium reached $[\mathrm{Fe} / \mathrm{H}] \approx-1.0$ dex, while the "accreted" objects subsequently formed the bulk of the Galactic halo. By this term we mean all of the objects that were born outside the single protogalactic cloud, i.e., in the nearest satellite galaxies or in isolated protogalactic fragments, and that subsequently escaped from them under the Galactic tidal forces.

Figure 2 shows the metallicity-relative magnesium abundance diagram for our catalog, where different symbols denote stars belonging to the accreted halo, to the protodisk halo, and to both disk subsystems. We see from the figure that the relative magnesium abundances in protodisk halo stars are virtually independent of metallicity and that all stars of this halo lie above the dashed line drawn through $[\mathrm{Mg} / \mathrm{Fe}]=0.2$ dex i.e., the relative magnesium abundances in protodisk halo stars are higher than those in the bulk of the thin-disk stars (Marsakov and Borkova 2005). One cannot but notice the presence of an appreciable number (five) of relatively metal-rich $([\mathrm{Fe} / \mathrm{H}]>-1.4 \mathrm{dex})$ stars with high relative magnesium abundances $([\mathrm{Mg} / \mathrm{Fe}]>0.5 \mathrm{dex})$ in the diagram; no theoretical models of chemical evolution predict their appearance. The impression is that when a metallicity $[\mathrm{Fe} / \mathrm{H}] \approx-1.5$ dex is reached in the interstellar medium more massive SNe II begin to explode in it. Given the lack of data on such stars in our sample, we will not consider this point here. The remaining protodisk halo stars lie in a narrow strip in the diagram in good agreement with the theoretically predicted dependence (see, e.g., the model by Matteucci and Francois (1989)). This behavior of protodisk halo stars suggests that, at least in the initial stage of its formation the interstellar matter in the early Galaxy either was well mixed or SNe II of the same mass exploded in all local volumes. In contrast, the presumably accreted stars exhibit a large spread in relative magnesium abundances in Fig. 2 that extends to negative $[\mathrm{Mg} / \mathrm{Fe}]$. The anomalously low relative magnesium abundances in some of the accreted stars are usually explained by an extremely low star formation rate in the dwarf satellite galaxies where these stars were born (Gilmore and Wise 1998). However our analysis of the relative magnesium and europium abundances in a small sample of nearby field stars showed that all of the presumably accreted stars exhibited an $[\mathrm{Eu} / \mathrm{Mg}]$ ratio that differed sharply from its Galactic value (Borkova and Marsakov 2004). Since the relative yield of these elements depends solely on the masses of the SN II progenitor stars where they are synthesized, we believe that a more likely mechanism of the magnesium abundance variations in accreted stars is the difference between the initial mass functions in their parent dwarf satellite galaxies. Therefore, it is interesting to try to identify genetically related stars in the accreted halo.

In the above paper we drew attention to a small group of stars with identical azimuthal velocities and pointed out that their orbital elements are in satisfactory agreement with those of the most massive Galactic globular cluster $\omega$ Centauri which is believed to have been the nucleus of a dwarf galaxy in the past (Larson 1996). Shortly after the appearance of our paper Meza et al. (2005) published their paper in which the authors used numerical simulations from Abadi et al. (2003) to investigate the orbital characteristic of dwarf satellite galaxies whose orbits were reduced significantly and dragged into the Galactic plane by dynamical friction even before their disruption. Disrupted on very eccentric orbits coplanar with the disc, such galaxies are expected to shed stars in "trails" of distinct orbital energy and angular momentum during each pericentric passage. Therefore, to an 
observer located between the pericentre and apocentre of such orbits, these trails would show as distinct groupings of stars with low vertical velocities and a broad, symmetric, often double-peaked distribution of Galactocentric radial velocities. Based on the recommendations of Meza et al. (2005), we identified stars that were lost by the dwarf galaxy whose center was the cluster $\omega$ Cen by the azimuthal and vertical velocities in the ranges $-50 \leq \Theta \leq 0$ and $|W|<65 \mathrm{~km} \mathrm{~s}^{-1}$, respectively. There are 18 such stars in our sample. In the $[\mathrm{Mg} / \mathrm{Fe}]-[\mathrm{Fe} / \mathrm{H}]$ diagram (see Fig. 2), all of them (except one deviated star G 233-026) lie along a narrow strip that exhibits an approximately constant ratio of $[\mathrm{Mg} / \mathrm{Fe}] \approx 0.35 \mathrm{dex}$ at low metallicities $([\mathrm{Fe} / \mathrm{H}] \leq-1.3 \mathrm{dex})$. Subsequently however as the metallicity increases, the relative magnesium abundance decreases sharply to negative values starting from this point. This behavior closely resembles the expected $[\mathrm{Mg} / \mathrm{Fe}]-[\mathrm{Fe} / \mathrm{H}]$ relation derived in a closed model of chemical evolution (see, e.g., Matteucci and Francois 1989), which is independent evidence for the genetic relationship between the identified stars. (Meza et al. (2005) identified 11 members of this group and also pointed out the existence of a genetic relationship between them.) Hence, the low relative magnesium abundances in the metal-richest stars of this group resulted from the SN Ia explosions that began in their parent protogalactic cloud and that ejected a large number of iron atoms into the interstellar medium and reduced the $[\mathrm{Mg} / \mathrm{Fe}]$ ratio. The considerably lower metallicity of the knee point in this diagram than that in the Galaxy suggests that the stars of the Centaurus moving group were formed from matter in which the star formation rate was considerably lower than that in the early Galaxy. Hence, at least in this, presumably initially massive $\left(M \approx 10^{9} M_{\odot}\right.$; see Tshuchiya et al .2003) disrupted satellite galaxy the mean masses of the SN II progenitor stars were the same as those in our Galaxy.

Let us divide all our presumably accreted stars into four azimuthal velocity ranges by $\Theta=(-250,-50$, and +50$) \mathrm{km} \mathrm{s}^{-1}$. In the $(\Theta-[\mathrm{Mg} / \mathrm{Fe}])$ and $([\mathrm{Fe} / \mathrm{H}]-[\mathrm{Mg} / \mathrm{Fe}])$ diagrams, the stars of each range are plotted by different symbols (see Figs. $3 \mathrm{a}$ and $3 \mathrm{~b}$, respectively). The dashed lines separate the relatively magnesium-poor and magnesium-rich stars by the ratio dex. The overwhelming majority (12 of 16) magnesium-poor accreted stars of moderate metallicity $(-1.3 \mathrm{dex}<[\mathrm{Fe} / \mathrm{H}]<-0.7 \mathrm{dex})$ fell within the range $|\Theta|<50 \mathrm{~km} \mathrm{~s}^{-1}$ (the filled triangles in the diagram). (Note that there are also stars among these 12 stars that we attributed to the accreted halo, despite their residual velocities that did not reach the critical value; their numbers are given in Fig. 3b.) A test showed that the orbits of these 12 stars go away not very far from the Galactic plane: together with the four stars from the Centaurus group located here $\left\langle\left|Z_{\max }\right|\right\rangle=8.0 \pm 1.2 \mathrm{kpc}$ for them. At the same time, the mean largest distance of the orbital points from the Galactic plane for the remaining five stars (see Fig. $3 \mathrm{~b}$ ) that slowly revolve around the Galactic center but that are metal-poorer ones $([\mathrm{Fe} / \mathrm{H}]<-1.3 \mathrm{dex})$ is more than twice as large: $19.1 \pm 2.9 \mathrm{kpc}$. Thus almost all of the magnesium-poor stars of moderate metallicity are found to fall within the solar neighborhood from the satellite galaxies that began to lose their stars only after their orbits were strongly distorted by the gravitational interaction with the Galactic disk and were brought closer to its plane. Their masses must probably be high enough to resist the Galactic gravitational forces for a long time and to retain stars near them. The assumption that the masses of these satellites are fairly high is also con firmed by the fact that their chemical evolution is well interpreted in terms of the closed model. (Indeed, only at a mass of the protocloud above a certain limit is the enriched gas not swept out from it by supernova explosions.) In contrast, the disruption of less massive satellites began earlier; therefore, the orbits of the stars lost by them are generally higher. The low ratios $[\mathrm{Mg} / \mathrm{Fe}]<0.2$ dex at metallicities in the range -1.4 dex $<[\mathrm{Fe} / \mathrm{H}]<-2.4$ dex for the 
above ve slow but high-orbit magnesium-poor stars can hardly be explained by the low star formation rate in their parent dwarf galaxies alone. Here, a deficit (or complete absence) of massive $\left(M>20 M_{\odot}\right)$ SNe II which are the main suppliers of primary $\alpha$-element atoms, is most likely responsible for the low magnesium abundance.

Stars with large absolute values of the azimuthal velocities in the solar neighborhood also have large apogalactic radii of their orbits. Therefore, according to Abadi et al. (2003), they must come into the Galaxy predominantly from less massive and, hence, early disrupted dwarf galaxies in which there seems to have been no star formation after the first generation SNe Ia explosions. Let us examine the behavior of stars from the higher-velocity $\Theta$ ranges in the $[\mathrm{Fe} / \mathrm{H}]-[\mathrm{Mg} / \mathrm{Fe}]$ diagram. The stars from symmetric (in velocities), but different (in direction of rotation) ranges $\left(-250<\Theta<-50 \mathrm{~km} \mathrm{~s}^{-1}\right.$ and $\Theta>50 \mathrm{~km} \mathrm{~s}^{-1}$ ) are randomly distributed over the field of the diagram and reveal no clearly traceable sequences that would be indicative of a genetic relationship between some of them. However it may be noted that positively rotating stars are, on average, metal-richer and magnesiumpoorer $(\langle[\mathrm{Fe} / \mathrm{H}]\rangle=-1.47$ and -1.91 dex, while $\langle[\mathrm{Mg} / \mathrm{Fe}]\rangle=0.23$ and 0.34 dex respectively for positively and negatively rotating groups of stars). At the same time, all seven stars with the highest negative azimuthal velocities (the crosses in Fig. $3 \mathrm{~b}$ ) form a compact group with $\langle[\mathrm{Fe} / \mathrm{H}]\rangle=-1.68$ dex and a small spread, $\sigma_{[\mathrm{Fe} / \mathrm{H}]}=0.14$ dex which is almost equal to the error in metallicity and show anomalously low relative magnesium abundances $(\langle[\mathrm{Mg} / \mathrm{Fe}]\rangle=0.09 \pm 0.02 \mathrm{dex})$. Thus there are no more or less numerous groups with a common origin among the rapidly rotating accreted stars; therefore, all of them seem to have come into the solar neighborhood from various stellar ensembles. The most noticeable common property that we can point out for magnesium-poor stars so far is the displacement of the mean metallicity to the negative values for them when passing from the stars that revolve slowly around the Galactic center to those that revolve rapidly: $\langle[\mathrm{Fe} / \mathrm{H}]\rangle=-1.2 \pm 0.1$ dex and $-1.7 \pm 0.1 \mathrm{dex}$, respectively for the group with $|\Theta|<50 \mathrm{~km} \mathrm{~s}^{-1}$ and $\Theta<-250 \mathrm{~km} \mathrm{~s}^{-1}$.

Note that two thirds of our stars in the velocity range $|\Theta|>50 \mathrm{~km} \mathrm{~s}^{-1}$ have only one published relative magnesium abundance determination while within the range $|\Theta|<$ $50 \mathrm{~km} \mathrm{~s}^{-1}$ the $[\mathrm{Mg} / \mathrm{Fe}]$ ratios for half of the stars were averaged over several sources. As a result, more than one source is available only for $\approx 10 \%$ of the metal-poor $([\mathrm{Fe} / \mathrm{H}]<$ -1.0 dex $)$ magnesium poor $([\mathrm{Mg} / \mathrm{Fe}]<0.2$ dex $)$ stars. Therefore, it is desirable to confirm the results of this section by further observations.

\section{RELATION BETWEEN CHEMICAL COMPOSITION AND ORBITAL ELEMENTS OF STARS}

Let us analyze in more detail the relations between the chemical composition of accreted stars and their orbital elements. Figure $4 \mathrm{a}$ where only accreted halo stars are plotted in the $\Theta-[\mathrm{Mg} / \mathrm{Fe}]$ diagram shows more clearly the trend found in the previous section. We see that the lower limit for the relative magnesium abundance in accreted stars is almost independent of their azimuthal velocity. Since the right and left corners of the diagrams are totally devoid of stars, the upper envelope of the diagram is naturally described by two inclined lines that intersect near $\Theta \approx 0 \mathrm{~km} \mathrm{~s}^{-1}$. In other words the upper limit for the $[\mathrm{Mg} / \mathrm{Fe}]$ ratio in accreted stars systematically decreases with increasing absolute value of the azimuthal velocity. As a result, we see that accreted stars with high relative magnesium abundances tend to have low orbital velocities around the Galactic center at the solar Galactocentric distance. At the same time, stars with low $[\mathrm{Mg} / \mathrm{Fe}]$ ratios are 
distributed more uniformly in all permitted values.

Since the linear velocity of a star is known to depend its orbital phase, it is not surprising that the $\zeta-[\mathrm{Mg} / \mathrm{Fe}]$ diagram in Fig. $4 \mathrm{~b}$ where the orbital inclination is defined as $\zeta=$ $\arcsin Z_{\max } / R a$ and its sign is the same as that of the azimuthal velocity also shows a similar structure. In this diagram, all points can also be bounded by three straight lines that form a triangle. Here, however a straight line represents well the lower envelope only in the segment $|\zeta| \leq 45^{\circ}$ while there are no stars with such low magnesium abundances outside this range; the stars concentrate to $\zeta=0^{\circ}$ at any $[\mathrm{Mg} / \mathrm{Fe}]$. Thus the stars with orbits lying near the Galactic plane are found, first, to constitute the majority of accreted stars and, second, to exhibit a wider range of relative magnesium abundances in both directions.

In Figs. $5 \mathrm{a}$ and $5 \mathrm{~b}$, the $[\mathrm{Mg} / \mathrm{Fe}]$ ratios are plotted against the maximum distances of the orbital points of stars from the Galactic center and plane. Both diagrams shows highly significant correlations for accreted stars: at the same correlation coefficients $(r=0.3 \pm 0.1)$, the probability of chance occurrence of both correlations for the same number $(N)$ of objects is $P_{N} \ll 1 \%$. (To ensure the reliability of the results, we discarded the five most distant points in the diagrams that often determine the correlation itself.) The two gradients in relative magnesium abundances were found to be negative rather than positive, as for all of the genetically related stars in the Galaxy and nonzero above the $2.5 \sigma$ level. (Although this is not seen on the scale of Fig. $5 \mathrm{~b}$, all of the accreted stars in our sample with maximum distances of their orbital points from the Galactic plane less than $1 \mathrm{kpc}$ (13 stars) have very high relative magnesium abundances, $[\mathrm{Mg} / \mathrm{Fe}]>0.3$ dex.) The detected gradients are not related to the evolution of the Galaxy but only reflect the sizes of the orbits of the satellite galaxies, lying on which they lose their stars under the Galactic tidal forces. The sizes of the orbits for accreted stars and, hence, for their disrupted parent galaxies are found to increase, on average, with decreasing relative magnesium abundance in them. Note, however that the lower envelopes in the diagrams more likely have an opposite slope and the magnesium abundance even slightly increases with both $R a$ and $Z_{\max }$. In other words despite the higher on average, relative magnesium abundances in them, there are also the magnesium-poorest accreted stars among the stars with small sizes of their orbits.

We see from Figs. $5 \mathrm{c}$ and $5 \mathrm{~d}$ that the same stars exhibit neither radial nor vertical metallicity gradients. However almost all of the stars here also occupy triangular regions in the diagrams that, in addition to the vertical axis, are bounded by two straight lines (the dashed lines in the diagrams); the upper and lower envelopes have opposite slopes and converge near $[\mathrm{Fe} / \mathrm{H}] \approx-1.5$ dex. As a result, the mean metallicity at any distance from the Galactic center and plane remains approximately constant, showing zero gradients, while the spread in $[\mathrm{Fe} / \mathrm{H}]$ decreases with increasing distance. As with magnesium there are both metal-poorest and metal-richest accreted stars among the stars with the smallest sizes of their orbits. It can be seen from a comparison of the two upper and two lower diagrams in Fig. 5 that a nearly solar magnesium abundance and a moderate (for old stars) heavy-element underabundance $([\mathrm{Fe} / \mathrm{H}] \approx-1.5 \mathrm{dex})$ are characteristic of the most distant orbits.

Protodisk halo stars are also plotted in Fig. 5. Our sample includes only 22 such stars, i.e., considerably fewer than the accreted stars (108 stars). Since such a small number does not allow statistically significant conclusions about the gradients in this subsystem to be drawn, we do not consider in detail the properties of its stars in this paper.

In Fig. 6, magnesium and iron abundances are plotted against eccentricities and perigalactic radii of the orbits for stars of different Galactic subsystems. In all diagrams, the 
stars of the genetically related subsystems (the thin and thick disks and the protodisk halo) form clearly traceable sequences. The sequences that intersect with the sequences of genetically related stars at a certain angle can also be traced for accreted stars (the corresponding regression lines are drawn). All correlations for genetically related stars are highly significant $\left(r \approx 0.60 \pm 0.02\right.$ at $\left.P_{N} \ll 1 \%\right)$. In contrast, for accreted stars, only the correlations between their metallicity and orbital elements are highly significant (in both cases, $r=0.3 \pm 0.1$ at $P_{N} \ll 1 \%$ ), while the mean relative magabundance in them is essentially uncorrelated with these orbital elements. Stars of different genesis are grouped in the opposite parts of the diagrams; exactly one half of the accreted stars have very high eccentricities, $e>0.9$ and small perigalactic radii of their orbits, $R p<1 \mathrm{kpc}$. The mean apogalactic radii their orbits are also small $(16 \pm 1 \mathrm{kpc})$. The stars of the Centaurus moving group that came into our Galaxy from a disrupted, fairly massive dwarf galaxy have precisely such orbital elements. This is understandable: more massive disrupted systems leave a more distinct trace among the Galactic field stars.

\section{THE GALACTIC HALO POPULATIONS}

Thus the trends found above make it possible to present the following formation picture of the Galactic halo. The small relative number of protodisk halo stars suggests that the star formation rate in a contracting protogalactic cloud was considerably lower than that at later stages of its evolution. Nevertheless, the star formation was fairly intense, since the metallicity of the interstellar medium had managed to increase to $[\mathrm{Fe} / \mathrm{H}] \approx-1.0$ dex solely through SN II ejections before SN Ia explosions began. The comparatively small spread in relative magnesium abundances in the stars of this subsystem indicates that the interstellar medium was well mixed in this evolutionary period of the Galaxy. Accreted stars constitute the bulk of the Galactic halo. They came into the Galaxy from the nearest dwarf satellite galaxies. According to the numerical simulations of the heir of archical Galactic halo formation performed by Abadi et al. (2003), massive satellite galaxies are disrupted up and lose their stars en masse only after dynamical friction reduces significantly the sizes of their orbits and brings them virtually into the Galactic plane. In contrast, less massive satellite galaxies (or those with orbits almost perpendicular to the disk) are disrupted even before their orbits change appreciably under the Galactic tidal forces. As a result, the stars lost by galaxies of different masses must have different kinematic characteristics. The relations between the iron and magnesium abundances and orbital elements of presumably accreted stars found here confirm this hypothesis put forward theoretically solely from dynamical considerations. Indeed, the 18 stars of the socalled Eggen moving group that we identified according to kinematic criteria exhibit a $[\mathrm{Fe} / \mathrm{H}]-[\mathrm{Mg} / \mathrm{Fe}]$ sequence characteristic of genetically related stars. With a high probability these stars belonged in the past to a fairly massive satellite galaxy whose central nucleus was the globular cluster $\omega$ Cen. The position of the knee in relative magnesium abundance at $[\mathrm{Fe} / \mathrm{H}] \approx-1.3$ dex indicates that the star formation rate in this dwarf galaxy was lower than that in our Galaxy. The star formation in this galaxy continued so long that its metal-richest stars reached $[\mathrm{Mg} / \mathrm{Fe}]<0.0$ dex, i.e., a ratio that is even smaller than that for the Sun. (A lower star formation rate than that in the Galaxy may also have played a certain role.) However the low value of the maximum metallicity in the stars of the group (only $[\mathrm{Fe} / \mathrm{H}] \approx-0.7$ dex) points to the cessation of further star formation in the parent galaxy. The very small scatter of points in this sequence suggests that the interstellar medium is well mixed in it. At the same time, the mean ratio $\langle[\mathrm{Mg} / \mathrm{Fe}]\rangle \approx 0.35$ dex in the metal-poor stars of this group, 
which is equal to its Galactic value suggests that the mean SN II masses are comparable in both galaxies, since the yield of $\alpha$-elements depends mainly on the presupernova mass. In other words, the chemical composition of the stars in this former galaxy (and their orbital elements)indicate that it actually had evolved for a fairly long time (but less than our Galaxy) before disruption and the initial stellar mass function in it was the same as that in our Galaxy. The accreted stars of the sample with low azimuthal velocities $\Theta$ exhibit a similar pattern of relation between $[\mathrm{Fe} / \mathrm{H}]$ and $[\mathrm{Mg} / \mathrm{Fe}]$ : all metal-poor stars have relative magnesium abundances higher than those in several comparatively metal-rich stars for which the $[\mathrm{Mg} / \mathrm{Fe}]$ ratio reaches its solar value. The orbits of these stars proved to be smallest and most eccentric, as for the stars of the Centaurus group.

There is a considerable number of stars that are simultaneously poor in heavy elements $([\mathrm{Fe} / \mathrm{H}]<-1.0$ dex $)$ and in magnesium $([\mathrm{Mg} / \mathrm{Fe}]<0.2 \mathrm{dex})$ among the stars revolving around the Galactic center with higher velocities (both positive and negative), while all of the stars with the highest negative velocities have such anomalously low relative magnesium abundances for very metal-poor $([\mathrm{Fe} / \mathrm{H}] \approx-1.7 \mathrm{dex})$ stars. The orbits of most stars with $|\Theta|>50 \mathrm{~km} \mathrm{~s}^{-1}$ are generally less eccentric and lie almost completely beyond the solar Galactocentric distance. Such orbits are characteristic of early disrupted low mass dwarf satellite galaxies. Shortly after their formation such low-mass galaxies begin to lose not only stars, but also interstellar gas while crossing the Galactic plane. In view of the loss of interstellar matter the star formation in them ends fairly rapidly; therefore, we barely see any metal-rich stars among them and it is unlikely that the anomalously low $[\mathrm{Mg} / \mathrm{Fe}]$ ratios for such metal-poor accreted stars resulted solely from an extremely low star formation rate in their parent dwarf galaxies. Probably the main reason for this is that the initial stellar mass function in less massive dwarf galaxies is just truncated at the high masses. As a result, SNeII eject a smaller amount of light $\alpha$-elements into the interstellar medium and the $[\mathrm{Mg} / \mathrm{Fe}]$ ratios for the stars become anomalously low compared the stars of the same metallicity that are genetically related to the single protogalactic cloud.

As has already been noted above, we drew the same conclusion from the fact that the $[\mathrm{Eu} / \mathrm{Mg}]$ ratios for a significant fraction of the accreted stars from the then analyzed small sample are considerably larger than those for the Galactic stars. Moreover in their recently published paper Decauwer et al. (2005) explained the difference in the slopes of the correlations between the primary and secondary $\alpha$-elements in 21 mildly metal-poor stars by the fact that the stars exhibiting lower than average $[\alpha / \mathrm{Fe}]$ ratios are formed in low-mass protogalactic fragments that are unable to sustain the formation of very massive stars. The authors believe that even at a constant initial mass function low-mass clouds have a lower probability of forming very massive stars. Direct $\alpha$-element abundance determinations in stars of isolated dwarf galaxies also lead to a similar conclusion. Thus, for example, Tolstoy et al. (2003) investigated the chemical composition in 15 red giants from four nearby low-mass $\left(\approx 2 \times 10^{7} M_{\odot}\right)$, very diffuse dwarf galaxies without central nuclei (Sculptor Fornax, Carina and Leo I). Almost all of the stars (14 of 15) fell on the $([\mathrm{Fe} / \mathrm{H}]-[\alpha / \mathrm{Fe}])$ diagram in such a way as if they were born in a single protogalactic cloud, i.e., forming a rather narrow sequence that sinks from the point with coordinates $([\mathrm{Fe} / \mathrm{H}]-\approx 2.0 \mathrm{dex}$ and $[\alpha / \mathrm{Fe}] \approx 0.3 \mathrm{dex})$ to the point with $(-1.0 \mathrm{dex}$ and $-0.2 \mathrm{dex})$. Not counting the two magnesium poorest stars (which is quite reasonable, bearing in mind the low accuracy of determining the elemental abundances in giants), the remaining 20 stars fell within the region occupied by our accreted stars with high orbital velocities around the Galactic center. The authors suggest that such low relative $\alpha$-element abundances could result only in the case of low star formation rates and the absence of any contribution from very 
massive $\left(>15--20 M_{\odot}\right)$ stars to the chemical evolution.

The results of our previous paper (Borkova and Marsakov 2002), where we found an anticorrelation between the Galactocentric distances and masses of globular clusters of a presumably extragalactic origin can also serve as a further argument in favor of the above suggestion. As a result, here we can also discern a statistical trend: predominantly lowmass metal-poor globular clusters turn out to be in distant orbits. It appears that low-mass globular clusters are generally formed in low-mass dwarf galaxies.

Thus the five independent facts organically fit within the framework of a single hypothesis. According to this hypothesis, metal-poor stars with anomalously low $\alpha$-element abundances come into our Galaxy from debris of low-mass satellite galaxies in which the chemical evolution proceeded not only slowly but also in the absence of massive SNe II.

Note that all our conclusions were formulated on the basis of statistical analyses and are not absolute in nature. We can also give examples of observed deviations from the trend found. Thus for example, several low-mass globular clusters (Ter 7, Ter 8, Arp 2, Pal 12) with anomalously low $\alpha$-element abundances $([\alpha / \mathrm{Fe}]<0.1$ dex $)$ were formed in the currently disrupted very massive $\left(5 \times 10^{9} M_{\odot}\right.$; see Ibata and Rasoumov 1998) Sagittarius dwarf spherical galaxy to which at least 10 globular clusters belonged earlier (see Borkova and Marsakov (2004) and references therein). Since this galaxy is in a nearly polar orbit (Ibata et al. 1997), the globular clusters that escaped from it do not lie in the Galactic plane. (However according to Abadi et al. (2003), such an orbit would not experience the disturbing in uence of Galactic tidal forces.)

The abundances of the remaining $\alpha$-elements as well as $r$ - and $s$-process elements in the sample stars must be analyzed to reconstruct more accurately the formation history of the Galactic halo and to estimate the ratio of the contributions from slowed-down star formation and truncation of the upper limit for the stellar masses in low-mass dwarf galaxies that lead to the birth of stars with anomalously low relative magnesium abundances in them.

ACKNOWLEDGMENTS: We wish to thank the anonymous referee who found several inaccuracies in the manuscript. This work was supported in part by the Ministry of Science of Russia (project no.02.438.11.7001) and the Federal Agency for Education (projects RNP 2.1.1.3483 and RNP 2.2.3.1.3950).

\section{References}

[1] M.G. Abadi, M.G. Navarro, M. Steinmetzand, and V.R. Eke, Astrophys.J. 591, 499, (2003).

[2] C. Allen and A. Santillan, Rev.Mex.Astron.Astrophys. 22, 255 (1991).

[3] T.V. Borkova and V.A. Marsakov, Astron.Zh. 77, 750 (2000) [Astron.Rep. 44, 665 (2000)].

[4] T.V. Borkova and V.A. Marsakov, Bull.Spec.Astrophys.Obs. 54, 61 (2002).

[5] T.V. Borkova and V.A. Marsakov, Pis'ma Astron.Zh. 30, 173 (2004) [Astron.Lett. 30, 148 (2004)].

[6] T.V. Borkova and V.A. Marsakov, Astron.Zh. 82, 453 (2005) [Astron.Rep. 49, 405 (2005)]. 
[7] H. Decauwer, E. Jeh Cin, G. Parmentier and P. Magain, Astron. Astrophys. 433, 285 (2005).

[8] G. Gilmore and R.F.G. Wise, Astrophys.J. 367, L55 (1998).

[9] R.A. Ibata and A.O. Rasoumov, Astron. Astrophys. 336, 130 (1998).

[10] R.A. Ibata, R.F.G. Wyse, G. Gilmor et al. Astron.J. 113, 634 (1997).

[11] R.B. Larson, Astron.Soc.Pac.Conf.Ser. 92, 241 (1996).

[12] V.A. Marsakov and T.V. Borkova, Pis'ma Astron.Zh. 31, 577 (2005) [Astron.Rep. 31, $515(2005)]$.

[13] V.A. Marsakov and T.V. Borkova, Pis'ma Astron.Zh. 32, 419 (2006) [Astron. Lett. 32, 376 (2006)] (in press).

[14] V.A. Marsakov and A.A. Suchkov, Astron.Zh. 54, 1232 (1977) [Sov.Astron. 21, 700 (1977)].

[15] L. Mashonkina and T. Gehren, Astron.Astrophys. 376, 232 (2001).

[16] F. Matteucci, Nature, 414, 253 (2001).

[17] F. Matteucci and P. Francois, Mon.Not.R.Astron.Soc. 239, 885 (1989).

[18] A. Meza, J.F. Navarro, M.G. Abadi, and M. Steinmetz, Mon.Not.R.Astron.Soc. 359, $93(2005)$.

[19] K. Nomoto, K. Iwamoto, N. Nakasato et al. Nucl.Phys.A 621, 467 (1997).

[20] K.U. Ratnatunga, J.N. Bahcall and S. Casrtano, Astropys.J. 291, 260 (1989).

[21] F.-K. Thielemann, K. Nomotto, and G. Meyned, Astrophys.J. 460, 408 (1996).

[22] E. Tolstoy K.A. Venn, M. Shetrone et al., Astron.J. 125, 707 (2003).

[23] T. Tshuchiya, D. Dinescu, and V.I. Korchagin, Astrophys.J. 589, L29 (2003).

[24] T. Tsujimoto, K. Nomoto, Y. Yoshii, et al., Mon.Not.R.Astron.Soc. 277, 945 (1995). 

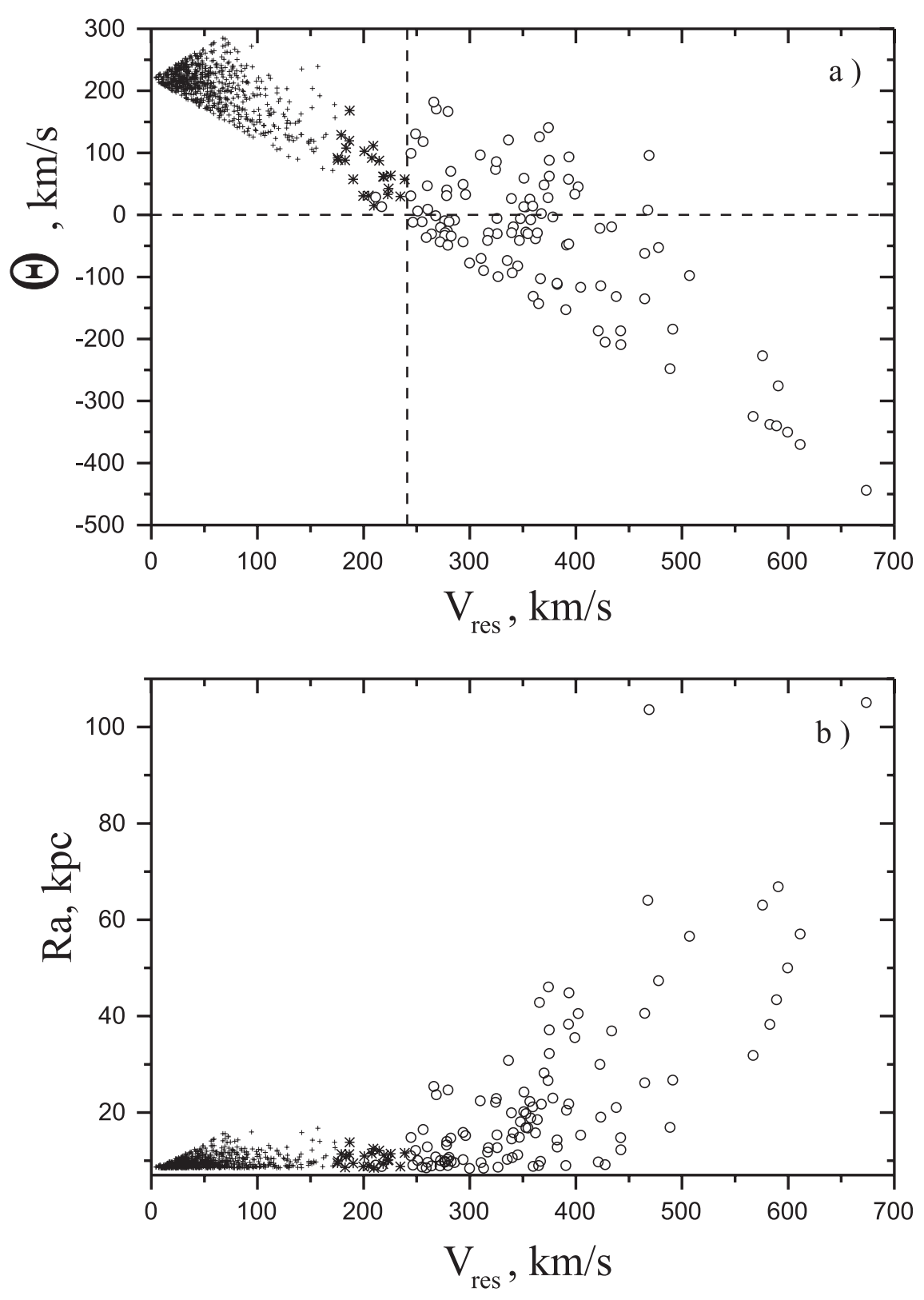

Figure 1: Residual stellar velocities with respect to the local standard of rest vs.(a) azimuthal velocities and (b) apogalactic orbital radii: the crosses, asterisks, and circles indicate thin-and thick-disk stars, protodisk halo stars, and presumably accreted stars, respectively. The horizontal dashed line separates the stars with prograde and retrograde orbits around the Galactic center while the vertical line separates the accreted stars from the stars genetically related to the Galaxy. 


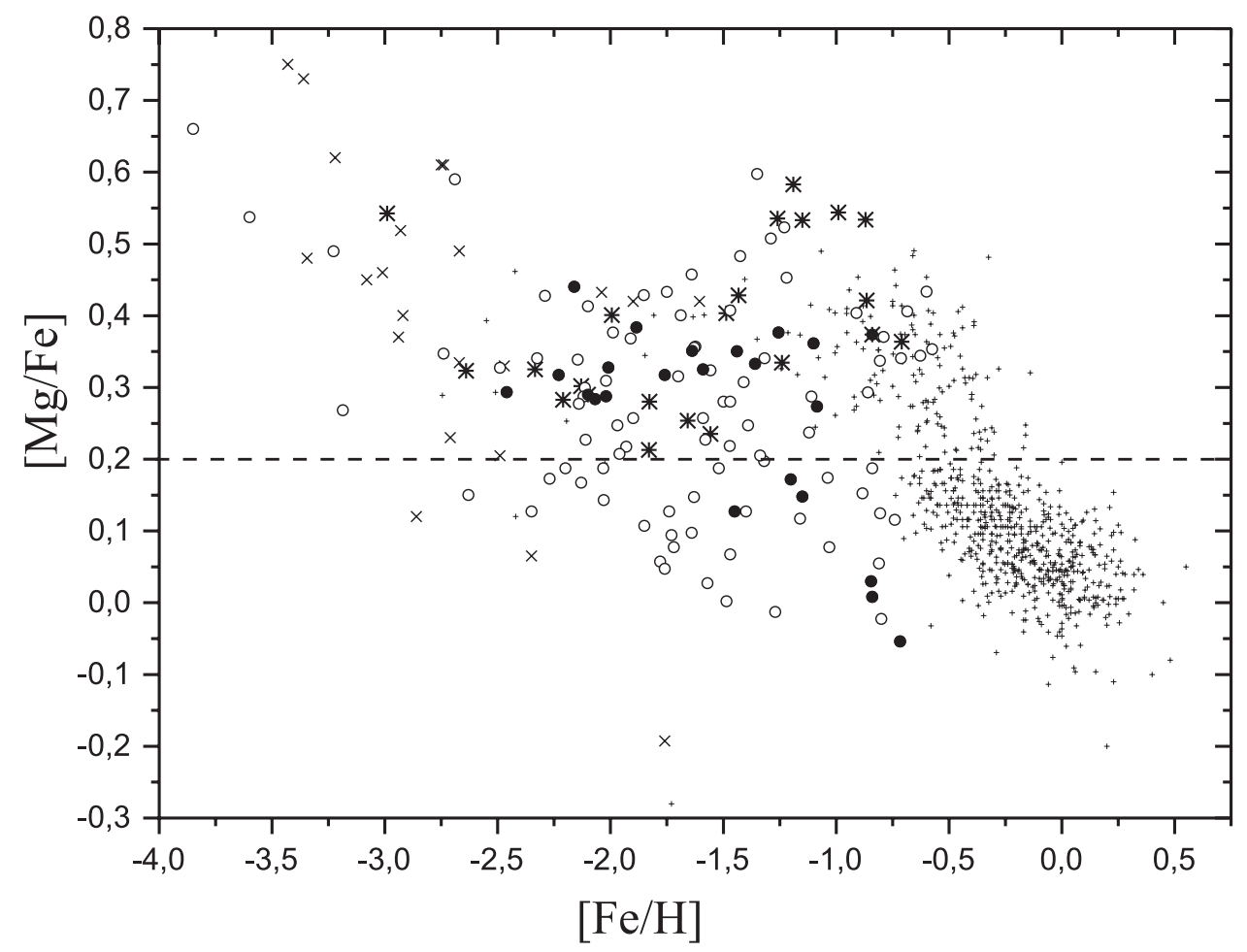

Figure 2: Metallicity vs. relative magnesium abundance for all of the stars in the catalog. The notation is the same as that in Fig. 1; the crosses indicate the stars that were not identified due to the lack of radial velocities. The dashed line was drawn through $[\mathrm{Mg} / \mathrm{Fe}]=0.2$ dex. The filled circles highlight the members of the Centaurus moving group among the accreted stars. 

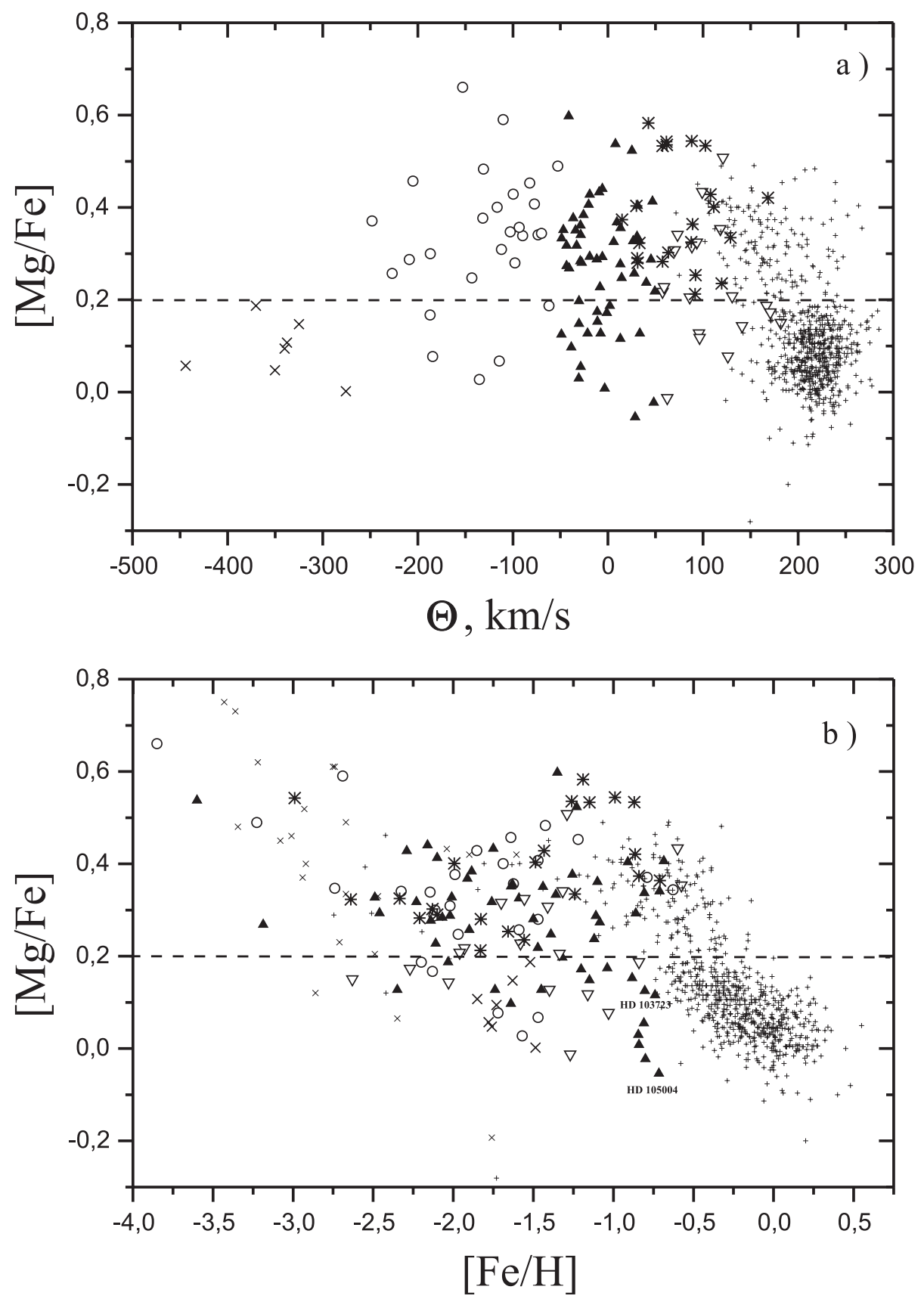

Figure 3: Relative magnesium abundances vs.(a) azimuthal velocities and (b) metallicity. The genetically related stars of the thin and thick disks and the protogalactic halo are denoted as in Fig. 1: the crosses, open circles, filled triangles with upward vortices, and open triangles with downward vortices represent presumably accreted stars with azimuthal velocities in the ranges $\Theta<-250,-250<\Theta<-50,-50<\Theta<+50$, and $\Theta>50 \mathrm{~km} \mathrm{~s}^{-1}$ respectively. 

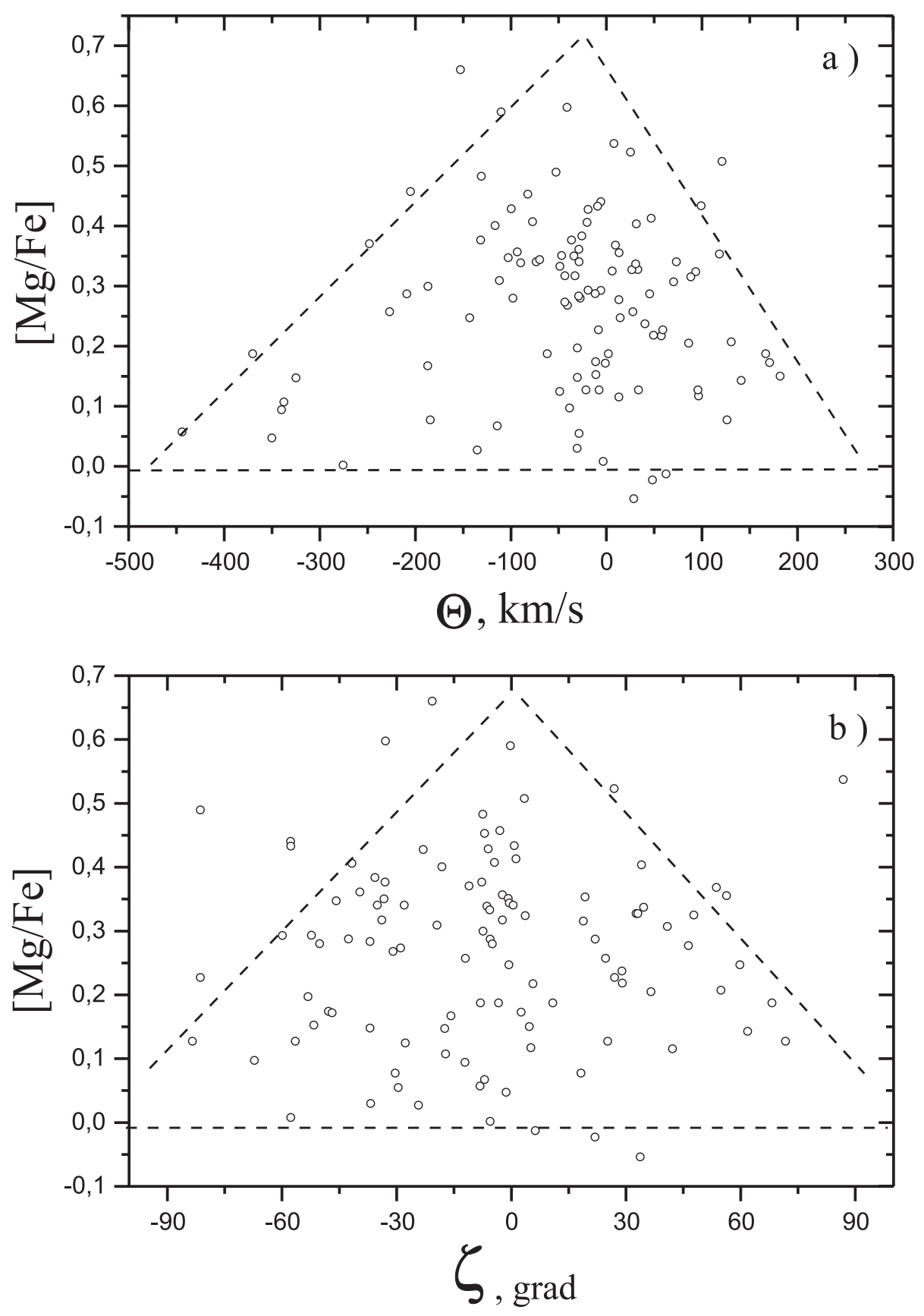

Figure 4: Relative magnesium abundances in accreted stars vs. (a) their azimuthal velocities and (b) Galactic orbital inclinations. The dashed lines represent the envelopes of the points in the diagrams drawn by eye. 

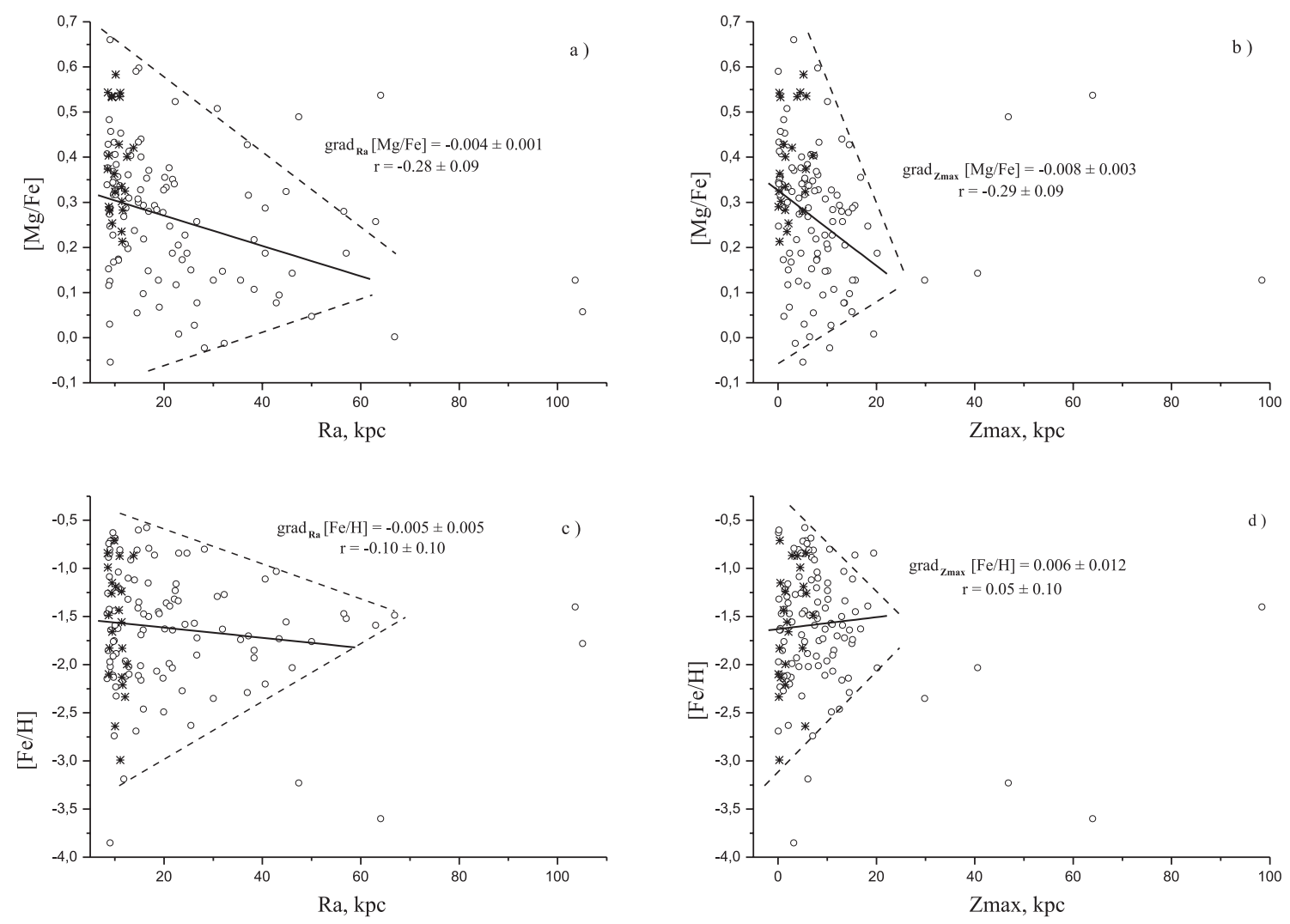

Figure 5: Relative magnesium abundances (upper row) and metallicities (lower row) in protodisk and accreted halo stars vs. maximum distances of the orbital points from the Galactic center (a, c) and plane (b, d). The notation is the same as that in Fig. 1. The solid lines represent the regression lines for accreted halo stars. The corresponding gradients and correlation coefficients are indicated. The dashed lines represent the lower and upper envelopes of the points in the diagrams drawn by eye. 

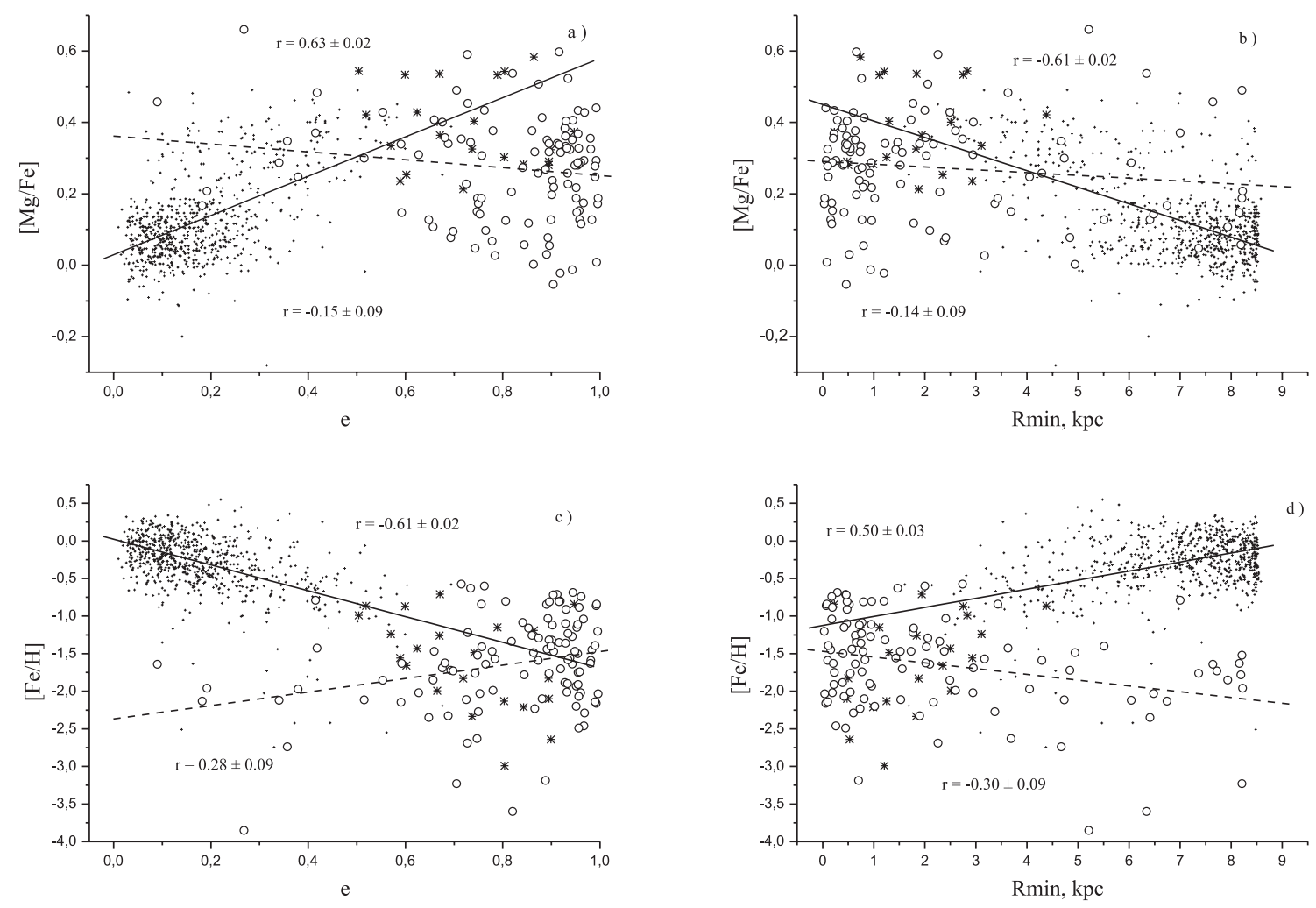

Figure 6: Relative magnesium abundances (upper row) and metallicities (lower row) in the stars of our catalog vs. eccentricities $(a, c)$ and perigalactic radii of their orbits (c,d). The notation is the same as that in Fig. 1. The solid and dashed lines represent the regression lines for genetically related stars and presumably accreted stars, respectively. The correlation coeffcients for genetically related stars and presumably accreted stars are given at the top and the bottom respectively. 